(c) Elsevier/INRA

Original article

\title{
A reduced animal model approach to predicting total additive genetic merit for marker-assisted selection
}

\author{
S Saito ${ }^{1}, \mathrm{H}$ Iwaisaki ${ }^{1,2^{*}}$ \\ ${ }^{1}$ Graduate School of Science and Technology; \\ ${ }^{2}$ Department of Animal Science, Faculty of Agriculture, \\ Niigata University, Niigata 950-21, Japan
}

(Received 22 February 1996; accepted 15 October 1996)

Summary - Using a system of recurrence equations, best linear unbiased prediction applied to a reduced animal model (RAM) is presented for marker-assisted selection. This approach is a RAM version of the method with the animal model to reduce the number of equations per animal to one. The current RAM approach allows simultaneous evaluation of fixed effects and total additive genetic merit which is expressed as the sum of the additive genetic effects due to quantitative trait loci (QTL) unlinked to the marker locus (ML) and the additive effects due to the QTL linked to the ML. The total additive genetic merits for animals with no progeny are predicted by the formulae derived for backsolving. A numerical example is given to illustrate the current RAM approach.

marker-assisted selection / reduced animal model / best linear unbiased prediction / total additive genetic merit / combined numerator relationship matrix

Résumé - Utilisation d'un modèle animal réduit pour prédire la valeur génétique globale dans la sélection assistée par marqueur. Sur la base d'un système d'équations de récurrence, la méthode du meilleur prédicteur linéaire sans biais appliquée à un modèle animal réduit (MAR) est présentée pour la sélection assistée par marqueur. Cette méthode est une version MAR de celle du modèle animal pour réduire à un le nombre d'équations par animal. Cette méthode MAR permet d'estimer simultanément les effets fixes et la valeur génétique globale, qui est la somme des effets génétiques additifs des locus de caractère quantitatif (QTL) non liés au locus marqueur et des effets additifs des QTL liés au locus marqueur. La valeur génétique globale des animaux sans descendance est prédite par un système d'équations reconstitué à partir du système principal. Un exemple numérique est donné pour illustrer la méthode MAR présentée ici.

sélection assistée par marqueur / modèle animal réduit / meilleur prédicteur linéaire sans biais / valeur génétique additive totale / matrice de parenté combinée

* Correspondence and reprints 


\section{INTRODUCTION}

Marker-assisted selection (MAS) is expected to contribute to genetic progress by increasing accuracy of selection, by reducing generation interval and by increasing selection differential (eg, Soller, 1978; Soller and Beckmann, 1983; Smith and Simpson, 1986; Kashi et al, 1990; Meuwissen and van Arendonk, 1992), especially for lowly heritable traits (Ruane and Colleau, 1995).

Fernando and Grossman (1989) presented methodology for the application of best linear unbiased prediction (BLUP; Henderson, 1973, 1975, 1984) to MAS in animal breeding. Using an animal model (AM) with additive effects for alleles at a marked quantitative trait locus (MQTL) linked to a marker locus (ML) and additive effects for alleles at the remaining quantitative trait loci (QTL) which are not linked to the ML, they showed the approach to simultaneous evaluation of fixed effects, effects of MQTL alleles, and effects of alleles at the remaining QTL. The number of equations required in the AM approach is $f+q(2 m+1)$ where $f, q$ and $m$ are the number of fixed effects, the number of animals in the pedigree file and the number of MQTLs, respectively. Therefore, the application of the AM approach may be limited to smaller data sets. Accordingly, Cantet and Smith (1991) derived a reduced animal model (RAM) version of Fernando and Grossman's approach, by which the total number of equations to be solved could be considerably reduced. The total additive genetic merit, ie, the sum of the value for polygenic effects and gametic effects can be predicted directly by an AM procedure (van Arendonk et al, 1994). The number of equations required in the procedure is $f+q$ since the number of equations per animal is reduced to one by combining information on the MQTL and the remaining QTL into one numerator relationship matrix.

BLUP methods for MAS require computation of the inverse of the conditional covariance matrix of additive effects for the MQTL alleles. Fernando and Grossman (1989) derived an algorithm to compute the inverse, which requires not only information on marker genotypes but also information on the parental origin of marker alleles. Wang et al (1995) extended Fernando and Grossman's work to situations where paternal or maternal origin of marker alleles can not be determined and where some marker genotypes are uninformative.

In this paper, a RAM approach to the prediction of total additive genetic merit is presented. The number of equations in the system for this RAM approach becomes of the order $f+q_{1}$ where $q_{1}$ is the number of parental animals. Also, a small numerical example is given to illustrate the current approach.

\section{THEORY}

In the derivations, one MQTL and one observation per animal are assumed for simplicity. The conditional covariance matrix between additive effects of the MQTL alleles, given the marker information, is based on the recursive equation which was presented by Wang et al (1995). 


\section{AMs for MAS}

An AM discussed by Fernando and Grossman (1989) is written as

$$
\mathbf{y}=\mathbf{X} \boldsymbol{\beta}+\mathbf{Z u}+\mathbf{Z P v}+\mathbf{e}
$$

where $\mathbf{y}$ is the $n \times 1$ vector of observations, $\boldsymbol{\beta}$ is the $f \times 1$ unknown vector of fixed effects, $\mathbf{u}$ is the $q \times 1$ random vector with the additive genetic effects due to QTL not linked to the ML, $\mathbf{v}$ is the $2 q \times 1$ random vector with the additive effects of the MQTL alleles, $\mathbf{e}$ is the $n \times 1$ random vector of residual effects, and $\mathbf{X}, \mathbf{Z}$ and $\mathbf{P}$ are $n \times f, n \times q$ and $q \times 2 q$ known incidence matrices, respectively. The expectation and dispersion matrices for the random effects are assumed to be

$$
\mathrm{E}\left[\begin{array}{l}
\mathbf{u} \\
\mathbf{v} \\
\mathbf{e}
\end{array}\right]=\left[\begin{array}{l}
\mathbf{0} \\
\mathbf{0} \\
\mathbf{0}
\end{array}\right] \quad \operatorname{Var}\left[\begin{array}{l}
\mathbf{u} \\
\mathbf{v} \\
\mathbf{e}
\end{array}\right]=\left[\begin{array}{ccc}
\mathbf{A}_{u} \sigma_{u}^{2} & \mathbf{0} & \mathbf{0} \\
\mathbf{0} & \mathbf{A}_{v} \sigma_{v}^{2} & \mathbf{0} \\
\mathbf{0} & \mathbf{0} & \mathbf{I} \sigma_{e}^{2}
\end{array}\right]
$$

where $\mathbf{A}_{u}$ is the numerator relationship matrix for the QTL not linked to the ML, $\mathbf{A}_{v}$ is the gametic relationship matrix for the MQTL, I is an identity matrix, and $\sigma_{u}^{2}, \sigma_{v}^{2}$ and $\sigma_{e}^{2}$ are the variance components for the additive genetic effects due to QTL unlinked to the ML, for the additive effects of the MQTL alleles and for the residual effects, respectively. The mixed model equations for equation [1] are

$$
\left[\begin{array}{ccc}
\mathbf{X}^{\prime} \mathbf{X} & \mathbf{X}^{\prime} \mathbf{Z} & \mathbf{X}^{\prime} \mathbf{Z P} \\
\mathbf{Z}^{\prime} \mathbf{X} & \mathbf{Z}^{\prime} \mathbf{Z}+\mathbf{A}_{u}^{-1} \alpha_{u} & \mathbf{Z}^{\prime} \mathbf{Z P} \\
\mathbf{P}^{\prime} \mathbf{Z}^{\prime} \mathbf{X} & \mathbf{P}^{\prime} \mathbf{Z}^{\prime} \mathbf{Z} & \mathbf{P}^{\prime} \mathbf{Z}^{\prime} \mathbf{Z} \mathbf{P}+\mathbf{A}_{v}^{-1} \alpha_{v}
\end{array}\right]\left[\begin{array}{c}
\boldsymbol{\beta}^{\circ} \\
\widehat{\mathbf{u}} \\
\widehat{\mathbf{v}}
\end{array}\right]=\left[\begin{array}{c}
\mathbf{X}^{\prime} \mathbf{y} \\
\mathbf{Z}^{\prime} \mathbf{y} \\
\mathbf{P}^{\prime} \mathbf{Z}^{\prime} \mathbf{y}
\end{array}\right]
$$

where $\alpha_{u}=\sigma_{e}^{2} / \sigma_{u}^{2}$ and $\alpha_{v}=\sigma_{e}^{2} / \sigma_{v}^{2}$.

On the other hand, the total additive genetic merit is expressed as the sum of the additive genetic effects due to QTL not linked to the ML and the additive effects of the MQTL alleles, or $\mathbf{a}=\mathbf{u}+\mathbf{P v}$. Then, as discussed by van Arendonk et al (1994), equation [1] can be written as

$$
\mathbf{y}=\mathbf{X} \boldsymbol{\beta}+\mathbf{Z a}+\mathbf{e}
$$

With the model [3], the variance-covariance structure for the total additive genetic merit $\mathbf{a}$ is given by

$$
\mathbf{A}_{a} \sigma_{a}^{2}=\mathbf{A}_{u} \sigma_{u}^{2}+\mathbf{P} \mathbf{A}_{v} \mathbf{P}^{\prime} \sigma_{v}^{2}
$$

where $\mathbf{A}_{a}$ is the combined numerator relationship matrix, and $\sigma_{a}^{2}\left(=\sigma_{u}^{2}+2 \sigma_{v}^{2}\right)$ is the variance component of the total additive genetic merit. Assumptions on the expectation and dispersion parameters for the random effects in the model [3] are then expressed as

$$
\mathrm{E}\left[\begin{array}{l}
\mathbf{a} \\
\mathbf{e}
\end{array}\right]=\left[\begin{array}{l}
\mathbf{0} \\
\mathbf{0}
\end{array}\right] \quad \operatorname{Var}\left[\begin{array}{l}
\mathbf{a} \\
\mathbf{e}
\end{array}\right]=\left[\begin{array}{cc}
\mathbf{A}_{a} \sigma_{a}^{2} & \mathbf{0} \\
\mathbf{0} & \mathbf{I} \sigma_{e}^{2}
\end{array}\right]
$$


As described by van Arendonk et al (1994), the mixed model equations are

$$
\left[\begin{array}{cc}
\mathbf{X}^{\prime} \mathbf{X} & \mathbf{X}^{\prime} \mathbf{Z} \\
\mathbf{Z}^{\prime} \mathbf{X} & \mathbf{Z}^{\prime} \mathbf{Z}+\mathbf{A}_{a}^{-1} \alpha_{a}
\end{array}\right]\left[\begin{array}{c}
\boldsymbol{\beta}^{\circ} \\
\widehat{\mathbf{a}}
\end{array}\right]=\left[\begin{array}{c}
\mathbf{X}^{\prime} \mathbf{y} \\
\mathbf{Z}^{\prime} \mathbf{y}
\end{array}\right]
$$

where $\alpha_{a}=\sigma_{e}^{2} / \sigma_{a}^{2}$.

\section{The proposed RAM approach for MAS}

The vectors $\mathbf{y}, \mathbf{u}$ and $\mathbf{v}$ in equation [1] can be partitioned as $\mathbf{y}=\left[\begin{array}{ll}\mathbf{y}_{p}{ }^{\prime} & \mathbf{y}_{o}{ }^{\prime}\end{array}\right]^{\prime}$, $\mathbf{u}=\left[\begin{array}{ll}\mathbf{u}_{p}{ }^{\prime} & \mathbf{u}_{o}{ }^{\prime}\end{array}\right]^{\prime}$ and $\mathbf{v}=\left[\begin{array}{ll}\mathbf{v}_{p}{ }^{\prime} & \mathbf{v}_{o}{ }^{\prime}\end{array}\right]^{\prime}$, respectively, where the subscripts $p$ and $o$ refer to animals with progeny and without progeny, respectively. Then Cantet and Smith (1991) discussed the RAM version of the model of Fernando and Grossman (1989).

In the AM given as equation [3], the vector $\mathbf{a}$ is partitioned as $\mathbf{a}=\left[\begin{array}{ll}\mathbf{a}_{p}{ }^{\prime} & \mathbf{a}_{o}{ }^{\prime}\end{array}\right]^{\prime}$, where $\mathbf{a}_{p}$ and $\mathbf{a}_{o}$ represent the total additive genetic merit for the parents and for the non-parents, respectively. With the similar idea used in $\mathbf{y}, \mathbf{X}, \mathbf{Z}$ and $\mathbf{e}$, the RAM of equation [3] can be written as

$$
\left[\begin{array}{l}
\mathbf{y}_{p} \\
\mathbf{y}_{o}
\end{array}\right]=\left[\begin{array}{l}
\mathbf{X}_{p} \\
\mathbf{X}_{o}
\end{array}\right] \boldsymbol{\beta}+\left[\begin{array}{cc}
\mathbf{Z}_{p} & \mathbf{0} \\
\mathbf{0} & \mathbf{Z}_{o}
\end{array}\right]\left[\begin{array}{l}
\mathbf{a}_{p} \\
\mathbf{a}_{o}
\end{array}\right]+\left[\begin{array}{l}
\mathbf{e}_{p} \\
\mathbf{e}_{o}
\end{array}\right]
$$

For the RAM, it is necessary that $\mathbf{a}_{o}$ is expressed as a linear function of $\mathbf{a}_{p}$. Then we utilize a system of recurrence equations, as follows

$$
\mathbf{a}_{o}=\mathbf{K} \mathbf{a}_{p}+\boldsymbol{\varphi}
$$

where $\mathbf{K}$ is a matrix relating $\mathbf{a}_{o}$ to $\mathbf{a}_{p}$ and is defined by

$$
\mathbf{K}=\operatorname{Cov}\left(\mathbf{a}_{o}, \mathbf{a}_{p}{ }^{\prime}\right)\left[\operatorname{Var}\left(\mathbf{a}_{p}\right)\right]^{-1}
$$

and $\boldsymbol{\varphi}$ is the vector of the residual effects. The vectors $\mathbf{a}_{p}$ and $\mathbf{a}_{o}$ are expressed as $\mathbf{a}_{p}=\mathbf{u}_{p}+\mathbf{P}_{p} \mathbf{v}_{p}$ and $\mathbf{a}_{o}=\mathbf{u}_{o}+\mathbf{P}_{o} \mathbf{v}_{o}$, respectively. Moreover, $\mathbf{u}_{o}$ and $\mathbf{v}_{o}$ can be represented by linear functions of $\mathbf{u}_{p}$ and $\mathbf{v}_{p}$, respectively (Cantet and Smith, 1991). The additive genetic effects due to QTL not linked to the ML of an animal can be described as the sum of the average of those of its parents and a Mendelian sampling effect, or

$$
\mathbf{u}_{o}=\mathbf{T} \mathbf{u}_{p}+\mathbf{m}
$$

where the matrix $\mathbf{T}$ has zero elements except for 0.5 in the column pertaining to a known parent, and $\mathbf{m}$ is a vector of the Mendelian sampling effects.

The relationship between $\mathbf{v}_{o}$ and $\mathbf{v}_{p}$ is written as

$$
\mathbf{v}_{o}=\mathbf{B} \mathbf{v}_{p}+\varepsilon
$$

where $\mathbf{B}$ is a matrix relating the additive MQTL effects of animals to those of parents, and $\boldsymbol{\varepsilon}$ is a vector of the segregation residuals. If the situations where the parental origin of marker alleles is not determined are considered, as discussed by Wang et al (1995), B contains at most four non-zero elements in each row. If $s$ 
and $d$ stand for the sire and the dam of animal $i$, respectively, in scalar notation equation $[10]$ is rewritten, as follows

$$
v_{i}^{1}=b_{i 1}^{1} v_{s}^{1}+b_{i 2}^{1} v_{s}^{2}+b_{i 3}^{1} v_{d}^{1}+b_{i 4}^{1} v_{d}^{2}+\varepsilon_{i}^{1}
$$

and

$$
v_{i}^{2}=b_{i 1}^{2} v_{s}^{1}+b_{i 2}^{2} v_{s}^{2}+b_{i 3}^{2} v_{d}^{1}+b_{i 4}^{2} v_{d}^{2}+\varepsilon_{i}^{2}
$$

where $v_{x}^{l}(l=1$ or 2 and $x=i, s$ or $d)$ are the corresponding elements of $\mathbf{v}_{o}$ and $\mathbf{v}_{p}$. The coefficients $b_{i k}^{l}(k=1,2,3$ or 4$)$ are the conditional probabilities that $Q_{i}^{l}$ is a copy of $Q_{p}^{m}(m=1$ or 2 and $p=s$ or $d)$, given the marker information, where $Q_{x}^{m}$ stands for the MQTL allele linked to the allele $M_{x}^{m}$ at the QTL (Wang et al, 1995). Also, $\varepsilon_{i}^{1}$ and $\varepsilon_{i}^{2}$ are the segregation residual effects.

Consequently, in equation [8] the vector corresponding to animal $i$ of $\mathbf{K}$ can be computed as

$$
\mathbf{k}_{i}{ }^{\prime}=\mathbf{A}_{a_{p}}^{-1}\left(\mathbf{A}_{u_{p}} \mathbf{t}_{i}{ }^{\prime} \sigma_{u}^{2}+\mathbf{I}_{p} \otimes \mathbf{1}^{\prime} \mathbf{A}_{v_{p}} \mathbf{q}_{i}{ }^{\prime} \mathbf{1} \sigma_{v}^{2}\right) \sigma_{a}^{-2}
$$

where $\mathbf{A}_{a_{p}}, \mathbf{A}_{u_{p}}$ and $\mathbf{A}_{v_{p}}$ are appropriate submatrices of $\mathbf{A}_{a}, \mathbf{A}_{u}$ and $\mathbf{A}_{v}$, respectively, $\mathbf{t}_{i}$ is the vector corresponding to animal $i$ of $\mathbf{T}, \mathbf{q}_{i}$ is the matrix corresponding to animal $i$ of $\mathbf{B}, \mathbf{1}$ is the vector $\left(\begin{array}{ll}1 & 1\end{array}\right)^{\prime}$ and $\otimes$ stands for the direct product operator.

Using equation [7] in equation [6] gives

$$
\left[\begin{array}{l}
\mathbf{y}_{p} \\
\mathbf{y}_{o}
\end{array}\right]=\left[\begin{array}{c}
\mathbf{X}_{p} \\
\mathbf{X}_{o}
\end{array}\right] \boldsymbol{\beta}+\left[\begin{array}{c}
\mathbf{Z}_{p} \\
\mathbf{Z}_{o} \mathbf{K}
\end{array}\right] \mathbf{a}_{p}+\left[\begin{array}{c}
\mathbf{e}_{p} \\
\mathbf{Z}_{o} \varphi+\mathbf{e}_{o}
\end{array}\right]
$$

and further equation [11] can be arranged as

$$
\mathbf{y}=\mathbf{X} \boldsymbol{\beta}+\mathbf{W} \mathbf{a}_{p}+\boldsymbol{\theta}
$$

For this model [12], the assumptions for expectations and dispersion parameters of $\mathbf{a}_{p}$ and $\boldsymbol{\theta}$ are given by

$$
\mathrm{E}\left[\begin{array}{c}
\mathbf{a}_{p} \\
\boldsymbol{\theta}
\end{array}\right]=\left[\begin{array}{l}
\mathbf{0} \\
\mathbf{0}
\end{array}\right] \quad \operatorname{Var}\left[\begin{array}{c}
\mathbf{a}_{p} \\
\boldsymbol{\theta}
\end{array}\right]=\left[\begin{array}{cc}
\mathbf{A}_{a_{p}} \sigma_{a}^{2} & \mathbf{0} \\
\mathbf{0} & \mathbf{R}
\end{array}\right]
$$

where the matrix $\mathbf{R}$ is expressed as

$$
\left[\begin{array}{cc}
\mathbf{I}_{p} \sigma_{e}^{2} & \mathbf{0} \\
\mathbf{0} & \boldsymbol{\Phi}+\mathbf{I}_{o} \sigma_{e}^{2}
\end{array}\right]
$$

and then the elements of $\Phi$ are calculated by

$$
\phi_{i, i}=\left(\sigma_{a}^{2}+F_{i} \sigma_{u}^{2}+2 f_{i} \sigma_{v}^{2}\right)-\mathbf{k}_{i} \mathbf{A}_{a_{p}} \mathbf{k}_{i}{ }^{\prime} \sigma_{a}^{2}
$$

and

$$
\phi_{i, j}=\mathbf{t}_{i} \mathbf{A}_{u_{p}} \mathbf{t}_{j}{ }^{\prime} \sigma_{u}^{2}+\mathbf{1}^{\prime} \mathbf{q}_{i} \mathbf{A}_{v_{p}} \mathbf{q}_{j}{ }^{\prime} \mathbf{1} \sigma_{v}^{2}-\mathbf{k}_{i} \mathbf{A}_{a_{p}} \mathbf{k}_{j}{ }^{\prime} \sigma_{a}^{2}
$$


If we denote $\boldsymbol{\Phi}+\mathbf{I}_{o} \sigma_{e}^{2}$ by $\mathbf{R}_{o}$, then the inverse matrix of $\mathbf{R}_{o}$ can be obtained, as follows

$$
\mathbf{R}_{o, i}^{-1}=\left[\begin{array}{cc}
\mathbf{R}_{o, i-1}^{-1} & \mathbf{0} \\
\mathbf{0} & 0
\end{array}\right]+\left(\phi_{i, i}+\sigma_{e}^{2}-\mathbf{s}_{i}{ }^{\prime} \mathbf{R}_{o, i-1} \mathbf{s}_{i}\right)^{-1}\left[\begin{array}{cc}
\mathbf{s}_{i} \mathbf{s}_{i}{ }^{\prime} & -\mathbf{s}_{i} \\
-\mathbf{s}_{i}{ }^{\prime} & 1
\end{array}\right]
$$

with $\mathbf{s}_{i}=\mathbf{R}_{o, i-1}^{-1} \mathbf{r}_{o, i}^{i-1}$, where $\mathbf{r}_{o, i}^{i-1}$ is the subvector corresponding to animal $i$ of $\mathbf{R}_{o}$, which contains elements for animals 1 to $i-1$.

Thus, the mixed model equations for equation [12] are written as

$$
\left[\begin{array}{cc}
\mathbf{X}^{\prime} \mathbf{R}^{-1} \mathbf{X} & \mathbf{X}^{\prime} \mathbf{R}^{-1} \mathbf{W} \\
\mathbf{W}^{\prime} \mathbf{R}^{-1} \mathbf{X} & \mathbf{W}^{\prime} \mathbf{R}^{-1} \mathbf{W}+\mathbf{A}_{a_{p}}^{-1} \sigma_{a}^{-2}
\end{array}\right]\left[\begin{array}{c}
\boldsymbol{\beta}^{\circ} \\
\widehat{\mathbf{a}_{p}}
\end{array}\right]=\left[\begin{array}{c}
\mathbf{X}^{\prime} \mathbf{R}^{-1} \mathbf{y} \\
\mathbf{W}^{\prime} \mathbf{R}^{-1} \mathbf{y}
\end{array}\right]
$$

\section{Backsolving for animals with no progeny}

The total additive genetic effects of animals with no progeny can be predicted from the following equations

$$
\widehat{\mathbf{a}_{o}}=\mathbf{K} \widehat{\mathbf{a}_{p}}+\widehat{\boldsymbol{\varphi}}
$$

where

$$
\widehat{\boldsymbol{\varphi}}=\left(\mathbf{I}_{o}+\boldsymbol{\Phi}^{-1} \sigma_{e}^{2}\right)^{-1}\left(\mathbf{y}_{o}-\mathbf{X}_{o} \boldsymbol{\beta}^{\circ}-\mathbf{K} \widehat{\mathbf{a}_{p}}\right)
$$

The inverse of $\boldsymbol{\Phi}$ can be obtained according to equation [14].

\section{EXAMPLE}

We use a small example data set including six animals, four animals having progeny and two animals with no progeny, as given in table I.

Table I. Example data with pedigree of six animals.

\begin{tabular}{lccccc}
\hline Animal & Sire & Dam & \multicolumn{2}{c}{ Genotype } & Observation \\
\hline 1 & - & - & $M_{1}$ & $M_{2}$ & 80 \\
2 & - & - & $M_{3}$ & $M_{4}$ & 120 \\
3 & 1 & 2 & $M_{1}$ & $M_{3}$ & 90 \\
4 & 1 & 2 & $M_{2}$ & $M_{3}$ & 110 \\
5 & 3 & 4 & $M_{3}$ & $M_{3}$ & 115 \\
6 & 3 & 2 & $M_{1}$ & $M_{3}$ & 100 \\
\hline
\end{tabular}

We assume $r=0.1$, where $r$ is the recombination rate between the ML and the MQTL. Then the gametic relationship matrix for the MQTL is as given in table II. The variance components assumed are $\sigma_{u}^{2}=0.3, \sigma_{v}^{2}=0.05, \sigma_{a}^{2}=0.4$ and $\sigma_{e}^{2}=0.8$. The incidence matrix $\mathbf{X}$ for fixed effects is assumed to be

$$
\mathbf{X}^{\prime}=\left[\begin{array}{llllll}
1 & 0 & 1 & 0 & 0 & 0 \\
0 & 1 & 0 & 1 & 1 & 1
\end{array}\right]
$$


Table II. The gametic relationship matrix for the additive effects of MQTL alleles.

\begin{tabular}{|c|c|c|c|c|c|c|c|c|c|c|c|}
\hline \multicolumn{2}{|c|}{1} & \multicolumn{2}{|c|}{2} & \multicolumn{2}{|c|}{3} & \multicolumn{2}{|c|}{$\begin{array}{c}\text { Animals } \\
4\end{array}$} & \multicolumn{2}{|c|}{5} & \multicolumn{2}{|c|}{6} \\
\hline $\mathrm{v}_{1}^{1}$ & $\mathrm{v}_{1}^{2}$ & $\overline{\mathrm{v}_{2}^{1}}$ & $\mathrm{v}_{2}^{2}$ & $\mathrm{v}_{3}^{1}$ & $\overline{\mathrm{v}_{3}^{2}}$ & $\mathrm{v}_{4}^{1}$ & $\mathrm{v}_{4}^{2}$ & $\overline{\mathrm{v}_{5}^{1}}$ & $\mathrm{v}_{5}^{2}$ & $\overline{\mathrm{v}_{6}^{1}}$ & $\mathrm{v}_{6}^{2}$ \\
\hline 1 & Sym. & $\begin{array}{l}0 \\
0 \\
1\end{array}$ & $\begin{array}{l}0 \\
0 \\
0 \\
1\end{array}$ & $\begin{array}{l}0.9 \\
0.1 \\
0 \\
0 \\
1\end{array}$ & $\begin{array}{l}0 \\
0 \\
0.9 \\
0.1 \\
0 \\
1\end{array}$ & $\begin{array}{l}0.1 \\
0.9 \\
0 \\
0 \\
0.18 \\
0 \\
1\end{array}$ & $\begin{array}{l}0 \\
0 \\
0.9 \\
0.1 \\
0 \\
0.82 \\
0 \\
1\end{array}$ & $\begin{array}{l}0.05 \\
0.05 \\
0.81 \\
0.09 \\
0.059 \\
0.819 \\
0.059 \\
0.819 \\
1\end{array}$ & $\begin{array}{l}0.05 \\
0.05 \\
0.81 \\
0.09 \\
0.059 \\
0.819 \\
0.059 \\
0.819 \\
0.666 \\
1\end{array}$ & $\begin{array}{l}0.81 \\
0.09 \\
0.09 \\
0.01 \\
0.9 \\
0.1 \\
0.162 \\
0.082 \\
0.135 \\
0.135 \\
1\end{array}$ & $\begin{array}{l}0 \\
0 \\
0.9 \\
0.1 \\
0 \\
0.82 \\
0 \\
0.82 \\
0.738 \\
0.738 \\
0.082 \\
1\end{array}$ \\
\hline
\end{tabular}

and the matrix $\mathbf{W}$ in equation $[12]$ is

$$
\mathbf{W}=\left[\begin{array}{llll}
1 & 0 & 0 & 0 \\
0 & 1 & 0 & 0 \\
0 & 0 & 1 & 0 \\
0 & 0 & 0 & 1 \\
-0.164 & 0.036 & 0.564 & 0.564 \\
0.002 & 0.402 & 0.58 & 0.016
\end{array}\right]
$$

The inverse matrix of $\mathbf{R}$ is given as

$$
\mathbf{R}^{-1}=\left[\begin{array}{llllll}
1.25 & 0 & 0 & 0 & 0 & 0 \\
& 1.25 & 0 & 0 & 0 & 0 \\
& & 1.25 & 0 & 0 & 0 \\
& & & 1.25 & 0 & 0 \\
\text { Sym. } & & & & 0.99375 & 0.00851 \\
& & & & & 1.01647
\end{array}\right]
$$

where $\mathbf{s}_{6}$ in equation [14] is -0.00837552 . Therefore, the coefficient matrix in equation [15] becomes

$$
\left[\begin{array}{llllll}
2.5 & 0 & 1.25 & 0 & 1.25 & 0 \\
& 4.52725 & -0.16232 & 1.69812 & 1.15977 & 1.83168 \\
& & 6.27673 & 2.49439 & -2.59154 & -2.5919 \\
& & & 6.4158 & -2.24072 & -2.47135 \\
& & & & 6.91362 & 0.32840 \\
& \text { Sym. } & & & & 6.56652
\end{array}\right]
$$


and the vector of right-hand side is

$$
\left[\begin{array}{llllll}
212.5 & 505.258 & 81.3235 & 195.4 & 236.958 & 204.077
\end{array}\right]^{\prime}
$$

Consequently, the vector of solutions for equation [15] is given as

$$
\left[\begin{array}{llllll}
85.9875 & 110.695 & -2.02273 & 2.02273 & 0.0477745 & 0.161347
\end{array}\right]^{\prime}
$$

and also, the vector of back-solutions in equation [16] is

$$
\left[\begin{array}{ll}
1.37642 & -1.34133
\end{array}\right]^{\prime}
$$

While the orders of the mixed model equations in the AMs of Fernando and Grossman (1989) and van Arendonk et al (1994) and in the RAM of Cantet and Smith (1991) are 20, 8 and 14, respectively, that in the current RAM approach is 6 , because animals 5 and 6 are non-parents. The solutions obtained by the current approach are the same as the corresponding ones calculated according to AMs of Fernando and Grossman (1989) and van Arendonk et al (1994).

\section{DISCUSSION}

For marker-assisted selection using BLUP, the AM approach was presented first by Fernando and Grossman (1989), and its RAM version was described by Cantet and Smith (1991). These AM and RAM approaches permit best linear unbiased estimation of fixed effects and simultaneous BLUP of the additive genetic effects due to QTL unlinked to the ML and the additive effects due to the MQTL.

On the other hand, van Arendonk et al (1994) discussed an AM method to reduce the number of equations per animal to one by combining information on MQTL and QTL unlinked to the ML into one numerator relationship matrix. Their method allows the prediction of only the total additive genetic merit in addition to the estimation of fixed effects. Accordingly, however, the size of mixed model equations required in their method can be smaller than those for the approaches by Fernando and Grossman (1989) and Cantet and Smith (1991).

The current approach is a RAM version of the method presented by van Arendonk et al (1994), and is given using a system of recurrence equations. In this RAM approach, the conditional covariance matrix for the MQTL can be computed by the method described by Wang et al (1995) which does not require assigning the origin of the marker alleles and accounts for inbred parents. With the current approach, there is a reduction expected in the size of mixed model equations since for the random effects only the equations for parental animals are required and the number of equations per parental animal is only one. However, one feature of the current method is that the matrix $\mathbf{R}$ defined in equation [13], essentially $\mathbf{R}_{o}=\boldsymbol{\Phi}+\mathbf{I}_{o} \sigma_{e}^{2}$, is not diagonal, and needs to be inverted before introduction into equation [15]. The computing algorithm shown in this paper could be one of the strategies for the practical calculation. Another feature of our approach is that sparseness in the coefficient matrix would be more destroyed, which could result in higher storage requirements. However, this may lead to easier convergence and 
reduction of computing time. Further comparisons between the current RAM and other approaches, for relative computational properties, are needed.

Hoeschele (1993) derived an AM approach considering equations for total additive genetic merits and additive effects due to the MQTL, where MQTL equations for animals not typed and certain other animals are absorbed. The method, for realistic situations, would also lead to a large drop in the number of equations required. A RAM consideration of Hoeschele's approach has been given by Saito and Iwaisaki (1996).

The BLUP methods for MAS, including the current RAM approach, require the knowledge of the recombination rate $(r)$ between the ML and the MQTL and the additive genetic variance explained by MQTL $\left(\sigma_{v}^{2}\right)$. Since true values of these parameters are usually unknown in practice, it is necessary that they are estimated. As discussed, eg, by Weller and Fernando (1991), van Arendonk et al (1993) and Grignola et al (1994), with the assumption of effects of MQTL alleles normally distributed, these parameters can be estimated by the likelihood-based methods such as restricted maximum likelihood (Patterson and Thompson, 1971).

\section{REFERENCES}

Cantet RJC, Smith C (1991) Reduced animal model for marker assisted selection using best linear unbiased prediction. Genet Sel Evol 23, 221-233

Fernando RL, Grossman M(1989) Marker assisted selection using best linear unbiased prediction. Genet Sel Evol 21, 467-477

Grignola FE, Hoeschele I, Meyer K (1994) Empirical best linear unbiased prediction to map QTL. In: Proceedings of the 5th World Congress on Genetics Applied to Livestock Production, University of Guelph, Ontario 21, 245-248

Henderson CR (1973) Sire evaluation and genetic trend. In: Animal Breeding and Genetics Symposium in Honor of Dr Jay L Lush, American Society of Animal Science and American Dairy Science Association, Champaign, IL, USA, 10-41

Henderson CR (1975) Best linear unbiased estimation and prediction under a selection model. Biometrics 31, 423-447

Henderson CR (1984) Applications of Linear Models in Animal Breeding. University of Guelph, Ontario

Hoeschele I (1993) Elimination of quantitative trait loci equations in an animal model incorporating genetic marker data. J Dairy Sci 76, 1693-1713

Kashi Y, Hallerman E, Soller M (1990) Marker-assisted selection of candidate bulls for progeny testing programmes. Anim Prod 51, 63-74

Meuwissen THE, van Arendonk JAM (1992) Potential improvements in rate of genetic gain from marker assisted selection in dairy cattle breeding schemes. J Dairy Sci 75, $1651-1659$

Patterson HD, Thompson R (1971) Recovery of inter-block information when block sizes are unequal. Biometrika $58,545-554$

Ruane J, Colleau JJ (1995) Marker assisted selection for genetic improvement of animal populations when a single QTL is marked. Genet Res Camb 66, 71-83

Saito S, Iwaisaki H (1996) A reduced animal model with elimination of quantitative trait loci equations for marker assisted selection. Genet Sel Evol 28, 465-477

Smith C, Simpson SP (1986) The use of genetic polymorphisms in livestock improvement. $J$ Anim Breed Genet 103, 205-217 
Soller M (1978) The use of loci associated with quantitative traits in dairy cattle improvement. Anim Prod 27, 133-139

Soller M, Beckmann JS (1983) Genetic polymorphism in varietal identification and genetic improvement. Theor Appl Genet 67, 25-33

van Arendonk JAM, Tier B, Kinghorn BP (1993) Simultaneous estimation of effects of unlinked markers and polygenes on a trait showing quantitative genetic variation. In: Proceedings of the 17th International Congress on Genetics, Birmingham, 192

van Arendonk JAM, Tier B, Kinghorn BP (1994) Use of multiple genetic markers in prediction of breeding values. Genetics 137, 319-329

Wang T, Fernando RL, van der Beek S, Grossman M, van Arendonk JAM (1995) Covariance between relatives for a marked quantitative trait locus. Genet Sel Evol $27,251-274$

Weller JI, Fernando RL (1991) Strategies for the improvement of animal production using marker-assisted selection. In: Gene Mapping: Strategies, Techniques and Applications (LB Schook, HA Lewin, DG McLaren, eds), Marcel Dekker, New York, USA, 305-328 